\title{
A theoretical framework for building the risk-resilience of basic infrastructures and services using Open Data
}

\author{
Mahsa Moghadas ${ }^{1, *}$, Alexander Fekete ${ }^{2}$, Asad Asadzadeh ${ }^{1}$ and Theo Kötter ${ }^{1}$ \\ ${ }^{1}$ Urban planning and land management group, Institute of Geodesy and Geoinformation, University of \\ Bonn/ Nussallee 1, 53115 Bonn, Germany \\ ${ }^{2}$ Institute for rescue engineering and civil protection, TH Köln - University of Applied Sciences, \\ Betzdorfer Straße 2, 50679 Cologne, Germany
}

E-Mails: m.moghadas@igg.uni-bonn.de; alexander.fekete@ th-koeln.de; asad.asadzadeh@ uni-bonn.de; tkoetter@uni-bonn.de

* Author to whom correspondence should be addressed; Tel.: +49-228-73-60359

\begin{abstract}
In recent years, cities have been confronted with increasing risks induced by climate change and natural hazards impacts. The experience gained highlights how cascading failures of critical infrastructures and basic services can affect both inherent and adaptive capacities of cities in time of an adverse event and create post disaster conflicts. At the same time, international policy circles have acknowledged the need to have sufficiently consistent and comparable disaster-related data to allow meaningful measurement of progress and impact. Therefore, to serve this need, understanding the link between resilience qualities of critical infrastructures and Open Data can lead to increase the level of disaster resilience, preparedness and response. It can also influence future-oriented urban and infrastructural planning at the local level. This paper offers a theoretical framework for building resilience in basic infrastructures and services across disaster preparedness and response concerning the contribution of Open Data for climate and natural hazard risk management in Tehran, Iran.
\end{abstract}

Keywords: Disaster Resilience; Basic Infrastructures and Services; Open Data

\section{Introduction}

Over the past ten years, approximately 700 thousand people have lost their lives, over 1.4 million have been injured and 23 million have been made homeless because of disasters partly fueled by climate change. One of the most widespread impacts of climate change is the increased frequency and severity 
of urban flooding because of intense rainfall. Based on the fact that the degree of exposure to flood as well as the inherent characteristics (vulnerability and existing capacitates) of the receptors such as human settlements, critical infrastructures and any other structure can affect the level of disaster risk, the need to strengthen cities' resilience have been acknowledged from global to local governance.

According to UNISDR resilience has been defined as "The ability of a system, community or society exposed to hazards to resist, absorb, accommodate, adapt to, transform and recover from the effects of a hazard in a timely and efficient manner, including through the preservation and restoration of its essential basic structures and functions through risk management".

Moreover, the role of critical Infrastructure in urban resilience to any kind of disaster is crucial because it provides the essential services and forms the backbone of cities. According to the UNISDR critical infrastructures refers to "The physical structures, facilities, networks and other assets which provide services that are essential to the social and economic functioning of a community or society". The New Urban Agenda by UN Habitat II highlighted that critical infrastructures and basic services consist of "delivery of safe water, sanitation, waste management, social welfare, transport and communication facilities, energy, health and emergency services, schools, public safety, and the management of open spaces". Likewise, Sustainable Development Goals (SDGs) especially goals 9 and 11 underscore the need for safe, resilient, and inclusive urban infrastructures with the ambition to "develop quality, reliable, sustainable, and resilient infrastructure in order to support community wellbeing with focus on affordable and equitable access for all".

There have been various attempts to address the challenges faced by urban development, climate change and disaster risk losses at the international level. One of the significant and complex challenges is that we need data to ensure that we are building resilient communities and infrastructures, which are inherently dynamic, with an effective monitoring system. Open Data is "digital data that is made available with the technical and legal characteristics necessary for it to be freely used, reused, and redistributed by anyone, anytime, anywhere". The approach that the data can be shared, collected and utilized as well as the quality of data can directly affect the level of risk information, disaster preparedness and respond and thus, improve outcomes for cities. Open Data helps governments to share, collect, and use data collaboratively in building urban resilience. Building resilient communities is a people-centered practice in which offering open-source tools can increase community engagement.

Despite the fact that Sendai Framework puts a strong emphasis on transparency, accountability, and the promotion of data collection, analysis, management and use by relevant users, Sendai Framework Data Readiness Review (2017) identified the scope of the gaps in disaster-related data availability, accessibility, quality, applicability and the "need to be sufficiently consistent and comparable to allow meaningful measurement of progress and impact". The identified gaps are not restricted to disaster loss data; statistical datasets are also in short supply, for example for measuring certain indicators. Therefore, local solutions for such challenge in disaster risk reduction and urban resilience at the national level is necessary.

Therefore, the key questions are firstly how Open Data, such as OpenStreetMap which is one of the most popular example of by Volunteered Geographic Information, can fill the gaps in resilience initiatives. Secondly, how community involvement e.g. the volunteer mappers for data creation and data utilization can help the local government dealing with disaster preparedness and response in a timely and efficient manner. Lastly, how this approach can help the decision makers for developing resilience policies and practices in Tehran, Iran. 


\section{Method}

This study is part of an ongoing $\mathrm{PhD}$ in the scope of INCOR Project in Iran that intends to develop a toolbox for urban resilience in order to address the local contribution to international agendas and reduce disaster losses by focusing on Basic Infrastructures and services (BIS) and the contribution of Open Data in disaster risk reduction for Tehran, the capital of Iran.

Tehran has a population of 8.43 million, and 13.6 million in the greater metropolitan area. Tehran is ranking at $10^{\text {th }}$ position within metropolitan areas at risk from earthquakes and river floods. In light of the fact that this mega city is vulnerable to flash floods from surface water or from the overflow of rivers caused by extreme rainfall, resilience assessment BIS can contribute to the overall resilience level of the city. Moreover, for such a dynamic megacity evolving, access to the real-time data is crucial in the context of flooding.

Table 1. The overview of the research project

Goal: Strengthening the resilience of BIS against flooding in Tehran using Open Data

\section{Objectives:}

- Quantifying BIS resilience dimensions

- Reviewing of contribution of Open Data in Disaster Risk Reduction

- Reduce BIS failure probabilities and its consequences through participatory approach

- Using Volunteered Geographic Information for creating real-time data for disaster response

Stakeholders: Universities, technical organizations, research centers and local government

\section{Method:}

- Develop a resilience assessment model (indicator based analysis)

- Conducting workshops for community volunteering building and training, leading to Crowdsourcing Geographic Knowledge through a participatory approach

- Collect the structured data of BIS elements and digitizing them in OpenStreetMap

- Create a comprehensive map of BIS and analyzing the level of resiliency based on flooding exposure map, spatial characteristics and the inherent capacities of them.

\section{Results:}

- Contributions to the Sendai Framework and increasing the opportunities for Sendai reporting

- Tehran has real-time data and an updated map for BIS, which leads to a better disaster preparedness and more effective disaster response

- Utilizing the data creation after the disaster results in an adaptive urban and infrastructural planning 


\section{Theoretical framework}

A framework is suggested in this paper to understand the qualities of risk and resilience for BIS and leverage community collaboration through Open Data in using, sharing and collecting consistent data which has been adopted from OpenDRI. Open Data includes a three-stage process of sharing data for increasing the public access to risk information; collecting data by community involvement; and using data by decision makers in disaster preparedness and response phase.

Moreover, we adopted the World Bank (2014 and 2015) qualities of urban resilience as a systems thinking approach, listed as inclusive, which refers to jointly vision to build resilience system and equitable access to BIS; redundant, which refers to spare capacity purposively created to accommodate disruption; reflective, which focuses on leveraging the past learning to inform and evolve future decisions; and robust, which refers to well-conceived, constructed and managed system. The set of indicators for each dimension can be defined accordingly. These qualities will be used as a basis for mapping the three main elements of critical infrastructures and their basic services including road networks and the related services such as emergency evacuation and logistics; hospitals and their services e.g. sanitation and temporary shelter; and schools and their secondary functions such as temporary shelters and temporary health centers. Figure 1 shows the theoretical framework of this study.

Figure 1. (a) Theoretical framework: Open Data for BIS

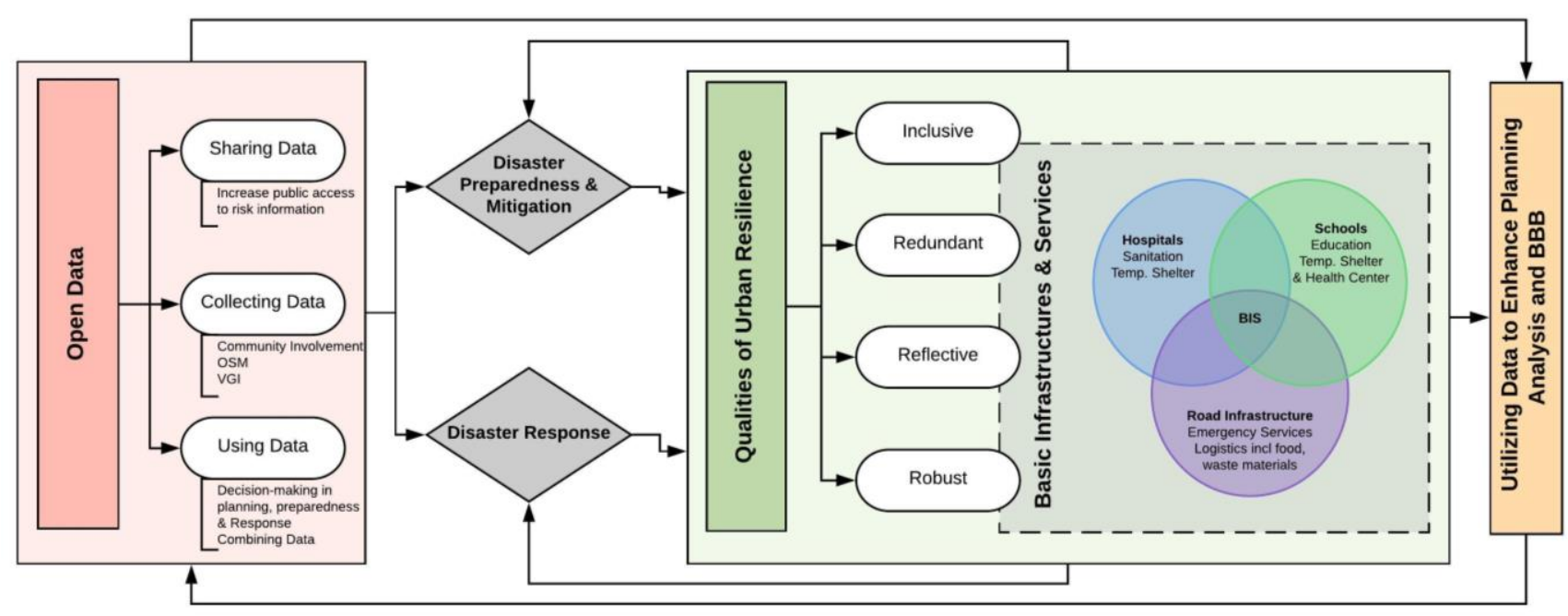

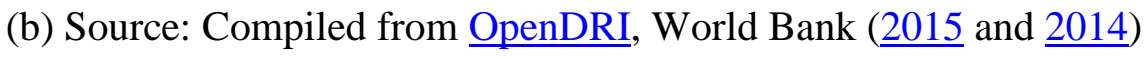

\section{Conclusion}

In urban resilience in general and in BIS resilience in particular against natural disasters, there is a widespread challenge in inconsistent and missing data as well as disaster-related data availability, accessibility and applicability. So that, there is a need for a collaborative and participatory approach for overcome this challenge. Open Data, crowdsourcing knowledge and Volunteered Geographic Information can be a tool to overcome this challenge in disaster management.

This study aimed to develop a theoretical framework for identifying the process of the research and illuminate the relationships between resilience contributing dimensions and BIS, in conjunction with the Open Data. On one hand, the four qualities of resilience are considered for BIS are inclusiveness, 
redundancy, reflectiveness, and robustness. These qualities can define flood resilience of BIS leading to quantitative measures in order to enhance their resilience level. On the other hand, Open Data which can serve urban resilience efforts, includes a three-stage process of sharing data $\mathrm{f}$, collecting data, and using data. This can help the government and decision makers to advance their capacity in disaster preparedness and act quickly in disaster response with available resources. There available resources are also the outcome a collaborative and participatory approach by community engagement, which results in improving of environmental risk information and communication for hazard risk reduction.

All in all, the resilience is about surviving and thriving, the process of data utilization not only can help the local government dealing with disaster preparedness and response in a timely and efficient manner, but also can influence future-oriented urban and infrastructural planning as well as Build Back Better. 